\title{
Genotype-Phenotype Databases: \\ Challenges and Solutions For The Post-Genomic Era
}

\author{
Gudmundur A Thorisson(1), Juha Muilu(2), Anthony J Brookes(1)*
}

(1) Department of Genetics, University of Leicester, University Road, Leicester, LE1 7RH, UK.

(2) Institute for Molecular Medicine Finland, University of Helsinki, Haartmaninkatu 8, FIN-00290, Helsinki, Finland.

* Corresponding Author:

Tel: +44 (0)116 2523401

Fax: +44 (0)116 2523378

Email: ajb97@leicester.ac.uk 


\section{Abstract}

Today's flow of research data concerning the genetic basis of health and disease is rapidly increasing in speed and complexity. In response, many projects are seeking to ensure that there are appropriate informatics tools, systems, and databases available to manage and exploit this flood of information. Previous solutions such as central databases, journal based publication, and manually intensive data curation are now being enhanced with new systems for federated databases, database publication, and more automated management of data flows and quality control. Along with emerging technologies that enhance connectivity and data retrieval, these advances should help to create a powerful knowledge environment for genotype-phenotype information. 
The World Wide Web has become an indispensable tool for biomedical researchers striving to understand how genes cause disease. Websites such as the PubMed literature search service ${ }^{1}$, the Ensembl ${ }^{2}, \mathrm{UCSC}^{3}$ and $\mathrm{NCBI}^{1}$ genome browsers, and the $\mathrm{BLAST}^{1}$ sequence search service, are examples of the internet resources that many biologists use on an almost daily basis. Behind the scenes these resources are based upon very similar technologies and design principles (i.e., they have standard 'architectures'), but their user-interfaces differ widely in terms of style, functionality and content. This diversity complements the diverse needs of the field, but to investigate a given biological question a user may need to browse many websites and still never feel sure they've tracked down all the information they might need.

While the proliferation of data resources can be frustrating for traditional biologists it presents an even bigger challenge for 'omics' researchers who need to automate largescale data aggregation across many different sites. Historically, such users were forced to write software to automatically surf websites to extract information originally designed for human consumption. As noted by $\mathrm{Stein}^{4}$, this 'screen scraping' approach has numerous disadvantages. Instead, there are better ways to inter-connect large sets of related information so that they can be searched and downloaded from a single portal.

In this review, we consider how this has been tackled in the past for genotype-tophenotype (G2P) data, and look at how the relevant technologies are currently being improved. We discuss some of the technical issues surrounding database development, and the recent trend towards an increased emphasis upon federated database solutions, which can link independent databases through a central portal and be married with the proven benefits of traditional central databases in which related data is stored all in one place. Looking further into the future, we consider even more revolutionary approaches to data integration and utilization, and discuss potential challenges that need to be addressed.

\section{Lessons from the past}

To understand the obstacles and the opportunities surrounding modern G2P databases, it is helpful to consider how the field has grown and evolved into its current state. Until recently, online stores of genetic data tended to be built as 'central databases' (Figure 1 ), and this model has served the field very well given its previous requirements.

Sequence Databases. The earliest databases of prominence in genetics were designed to hold DNA sequence data. In the early 1980 s, as soon as the use of commercial technologies for DNA sequencing became widespread, such depositories were needed to facilitate exchange and comparison of DNA sequences. Three major central databases were constructed for this purpose in Japan, the USA, and Europe; the $\mathrm{DDBJ}^{5}$, GenBank ${ }^{6}$ and EMBL ${ }^{7}$ respectively. In the mid 1980s the International Nucleotide Sequence Database Collaboration (INSDC, http://www.insdc.org) was established to promote full content exchange between these databases 
The INSDC constitutes a large-scale central database project, and it provides an excellent example of how effective the central databasing strategy can be. Arguably, however, this project succeeded as well as it did because: DNA sequence information is simple to represent as a directly annotated string of letters and sequence regions (i.e. it is ' 1 -dimensional'), and despite a massive growth in data volume, the scale of the problem did not exceed the capabilities provided by concomitant advances in computer technology.

Model Organism Databases. Model organism databases (MODs) provide a second example of how the central database model can be used effectively. These databases specialize in capturing genomic, phenotypic, and other information for a model organism. Examples include Wormbase ${ }^{8}$, the Rat Genome Database ${ }^{9}$ and the Mouse Genome Informatics Database ${ }^{10}$. A single or a few groups working closely together, armed with expert knowledge on their model organism of interest, were the typical creators of these early central G2P databases. The resulting websites provide a focal point for information gathering and access, plus centralised services such as a genome browser interface and tools for comparative genomics.

A simplistic assessment of MODs would put their success down to the very limited volume of data they have to manage, compared to the amounts flowing into a global nucleotide sequence database. But this view fails to allow for the fact that the data contained in an MOD are far more diverse and complex than mere 1-dimensional sequence strings (i.e., comprising genetic and phenotype related '2-dimensional' information). This complexity makes it far more difficult to organize the data within a single depository. The MODs overcame this hurdle by the virtue of good leadership and the relatively small community sizes that made it possible for agreements to be reached on matters such as data model standards, laboratory protocols, terminologies, and curation practices. Having these standards in place ensured that effective data management, interpretation and exchange could occur between the central database and many different laboratories. The MOD experience thus emphasizes the absolute need for robust and universal standards in order to aggregate and integrate G2P data. Extending this principle, MODs for many different species are now working together as part of the Generic Model Organism Database project (GMOD, http://www.gmod.org) to further harmonise and standardize their activities.

Central Databases for Human 'Mendelian Mutations'. The databasing of human G2P relationships has lagged behind what has been achieved in other species. There are many reasons for this, one of which is the far larger size and dispersed and diverse nature of the underlying research community that necessarily includes biologists, clinicians, epidemiologists, statisticians, etc. This has made it difficult to agree and deploy a full series of universal standards. Furthermore, the standards themselves are difficult to devise for human G2P relationships due to the complexity the data. These complexities include: the full spectrum of medical diagnoses and clinical test results that are often open to subjective interpretation; a wide range of normal traits that may vary with age and between populations; and a myriad of sequencing, genotyping and other 
laboratory procedures that are employed for the generation of primary data, which itself may be analysed and utilized in a plethora of different ways.

However, attempts have been made to capture a broad picture of human Mendelian G2P knowledge via the central database model. Good progress has been made by the Online Mendelian Inheritance in $\mathrm{Man}^{11}$ (OMIM:

http://www.ncbi.nlm.nih.gov/sites/entrez?db=omim) which provides a genotypephenotype catalogue of human genetic disorders, and which first appeared in book form over 40 years ago ${ }^{12}$. The project went online in 1990 and is now maintained by a substantial team of curators who manually extract experimental findings from the literature. This has resulted in a compilation of high-quality records on $\sim 11,000$ genes and diseases (as of April 1st, 2008). But this is a long way from being a fully comprehensive summary of all knowledge on human G2P relationships, even for Mendelian disorders. The task is simply too large for one team to manage in a centralized fashion, and given the complexity of the source information, OMIM is forced to package its content in narrative form. This makes it unsuitable for automated mining or deep integration with other database resources. Similar data collection issues are being faced by the Human Gene Mutation Database ${ }^{13}$ (HGMD: http://www.biobaseinternational.com/pages/index.php?id=hgmddatabase), which uses manual curation to extract from the literature a list of mutations that underlie Mendelian disorders, to then place these in a structured and readily searchable format. Another project with data collection challenges is the Pharmacogenetics and Pharmacogenomics Knowledge Base $^{14}$ (PharmGKB: http://www.pharmgkb.org) which collates extensive knowledge about the relationships amongst drugs, diseases and genes, to assist pharmacogenomics research. These examples illustrate some fundamental limitations, primarily relating to data complexity and quantity, to the central database concept, especially in the context of human G2P information.

Locus-Specific Databases for Human 'Mendelian Mutations'. Taking an alternative approach, many groups have collated primarily Mendelian G2P information for just one or a few genes of relevance to one or a few diseases of interest. The first of these 'Locus-Specific Databases' (LSDBs) was published in 1976, in the form of a catalog of human globin mutations ${ }^{15}$. Following a slow but steady increase in the number of online LSDBs, 700 are now listed at the Human Genome Variation Society website ${ }^{16}$ (http://www.hgvs.org/dblist/glsdb.html). LSDB entries tend to be rich in information content and enhanced by domain-specific expert curation. As well as published information they typically include unpublished DNA variation along with evidence concerning pathogenicity. Unfortunately, these databases are created independently of one another, with little coordination or harmonisation, and with little or no dedicated funding.

LSDBs range from a simple, non-networked spreadsheets through to fully-fledged online databases. Consequently they represent a fragmented network of silos full of rich information (Figure 2) across which it is not possible to efficiently exchange or integrate G2P information. In their current disjointed form, LSDBs are essentially at the opposite end of the spectrum from central G2P databases that provide shallower but genome- 
wide perspective. Neither is ideal, and so another approach - or combination of approaches - would seem to be needed.

\section{Challenges for Modern G2P Databases}

MODs, LSDBs and related databases have taught us that it will not be straightforward to computationally orchestrate the totality of human G2P information. Furthermore, the challenge is growing: high-throughput genomic data generation is now within the reach of many laboratory budgets. In addition it is now, or soon will be, possible to explore phenomena such as structural variation, DNA methylation, rare/unique alleles, and even somatic genome changes in a comprehensive manner.

Currently, genetic association studies, especially genome-wide association studies (GWAS) represent a particularly prolific source of G2P data. The principal databases set up to store and organize GWAS data include the dbGaP archive in the US ${ }^{17}$ (http://view.ncbi.nlm.nih.gov/dbgap), the EGA in Europe (http://www.ebi.ac.uk/ega), and the GWAS Database of Japan (https://gwas.lifesciencedb.jp/cgi-

bin/gwasdb/gwas top.cgi). Other related projects include HGVbaseG2P (http://www.hgvbaseg2p.org), GAD ${ }^{18}$ (http://geneticassociationdb.nih.gov), the Type 1 Diabetes Genetics Consortium (http://www.t1dgc.org), and disease-specific efforts AlzGene ${ }^{19}$ (http://www.alzforum.org/res/com/gen/alzgene), PDGene (http://www.pdgene.org), and SZGene ${ }^{20}$ (http://www.schizophreniaforum.org/res/sczgene/). At present, all of these employ the central database model, although it is unclear whether this approach will suffice in the longer term. As the field advances these databases will have to grapple with complex issues such as: increasingly convoluted data governance issues pertaining to different countries and legislatures; rapid changes in the scale, depth and format of the primary and processed data; and the likely addition of other forms of variation and levels of etiologic complexity. The over-riding need is to achieve a sufficient degree of global comprehensive coverage to make '2-dimensional' G2P databases as successful as '1dimensional' DNA sequence databases.

Database creators, their patrons, and their funding bodies are acutely aware of the need for more and better human G2P databases. Based on past experiences as elaborated above, and in light of recent technology developments, we suggest six key areas that need attention if improved G2P databases are to be built:

Data quantity: The scale of current and future G2P research means that datasets will keep getting larger and more numerous. This acceleration in the rate of data production might even start to outstrip the ability of database technologies to handle the information. For example, results may be repeatedly split and merged, and re-examined - as in the case of GWAS studies which share control materials and cross-compare their primary datasets, and which are being extended by further empirical work and by statistical re-analysis. The SNP data underlying GWAS results are also now being used 
to extract information on structural variation; and all of the above can be repeated adinfinitum for every phenotype and sub-phenotype characterized. The data volume will be further increased due to the addition of other forms of variation, other areas of bioscience, and the emergence of routine whole genome sequencing ${ }^{21}$. Therefore, data quantity must be a key consideration in database design.

Data quality: Even though database records will never be completely error free, efforts must be made to avoid inaccuracies wherever possible. Such activities can be split into manual data curation efforts that involve reading and redrafting of data that involves knowledge and concepts (e.g., from the literature into databases such as OMIM), versus automated validation of generally larger datasets with more straightforward content (such as markers, genotypes, and sequences). Quality control should be optimized right from the stage of data generation, but databases can only become involved from the stage of guiding researchers in the preparation of accurate and appropriate data submissions. Once data is received, databases should then deploy their own quality assurance measures to check for internal consistency and completeness of the submission. In scenarios where this requires manual curation, domain experts are invaluable, and they will often interact with the data submitters in performing their task. Future federated and community curation efforts (e.g., Wiki systems) will need to be carefully managed if they are to match the high standards achieved by current manual curation activities. The responsibility for that will lie, at least partly, with each database in the federated system, though oversight may be applied by stakeholders such as funders, international consortia, and feedback systems from the community as a whole. Today, databases obviously do try to ensure high data quality, and they know that this is a challenging business ${ }^{22}$, but perhaps in the future their obligations should go even further. For example, consistency with other data sources could be assessed; such as by comparing SNP allele frequencies with previous datasets to identify fundamental laboratory or data management errors, or cases where the wrong DNA strand has been referenced. Across the full breadth of G2P data there are many features that could be checked to ensure accuracy, and standards and guidance need to be developed to underpin data curation throughout the path of generating data through to placing it in public databases. Ideally, software support for this will increasingly be provided.

Data complexity: Although data quantity is a matter for concern, it will hopefully be overcome in time by improvements in data processing algorithms and innovations in computer science. More indomitable, however will be the matter of data complexity. Biological data, especially G2P data of the future, differs from that of most other 'big science' (such as astronomy) by its high-level of complexity ${ }^{23}$. Consider phenotypes for example: studies such as the UK Biobank (http://www.ukbiobank.ac.uk) and the Framingham Heart Study (http://www.framinghamheartstudy.org) collect thousands of phenotypic variables in a prospective manner, with each item supported by extensive metadata (information that describes the primary data), for tens or hundreds of thousands of subjects. The utilized phenotype definitions may change as knowledge advances, and patient phenotype categorizations may change with age and treatment. Given this data complexity, standardization of the 'phenotype' parameter is needed, and 
this is one of the goals of several projects, such as P3G ${ }^{24}$, HuGENet ${ }^{25}$, and the PhenX project (http://www.PhenX.org). The information that describes how genotypes connect to phenotypes - i.e., the ' 2 ' in G2P - is even more complex. A plethora of constantly evolving methods, strategies, and analyses that offer varying levels of precision, may be used to work out how DNA sequences control phenotypes. The results provide mere clues (sometimes of a contradictory nature) as to the underlying etiologic processes. Environmental effects (as being considered by the Genes, Environment and Health Initiative: http://www.gei.nih.gov), a person's genetic background, and chance, also feed into this. Thus, the complexity of the analytical methods and the experimental results make it difficult to store G2P information in a structured way, or to fully optimize the integration and presentation systems.

Knowledge representation: As more and more analyses are performed on ever more extensive and cross-domain datasets, it will become increasingly difficult to comprehensively gather and present all the resulting hypotheses and conclusions ('knowledge representation'). The issue of how to present conclusions is distinct from the question of what tools and systems are developed to generate those ideas, and how the systems interface with databases. Traditionally, scientific journals have been the principal vehicle for distributing the interpretation of data, but it not clear whether their current modus operandi will enable them to keep pace as the rate of new discoveries continues to grow exponentially. The human-readable narrative format of journals does not easily lend itself to the storage of 'interpretations' and 'concepts' in databases. However, some databases, such as OMIM, whose scope extends into knowledge representation, do employ free text. This resource illustrates the value of handling this kind of information beyond journals, but it also shows that it is limiting to store this data without any rigid structure. G2P knowledge representation therefore needs to become better structured and anchored upon appropriate ontologies if databases are to be more than just high-tech lists of primary experimental data.

Data access: As G2P datasets become larger and more diverse it will become increasingly difficult to locate any particular data item. To tackle this issue, more powerful tools for database searching will obviously be needed, but it is equally important that those improved search engines are connected to all the relevant data that needs to be searched. Although this could be seen as an argument for widespread adoption of the central database model, as detailed above, data size and complexity make it impossible to gather all the information in one central depository. Instead, complementary ways to consolidate the tasks of data access and data presentation across many different databases (e.g., LSDBs) are needed, such that the interrogated information itself never needs to leave its remote source. Such single point of access or federated database - solutions that tap into large volumes of diverse data are technically feasible. An example from outside the G2P domain is the ENCODEdb porta $^{26}$, which offers a simple query interface that searches across all the ENCODE experimental data deposited in several public databases.

Data sensitivity: Once the likely phenotypic consequences of carrying a particular sequence variation are established, knowing one's genotype at a given locus becomes 
meaningful. It also becomes meaningful for one's relatives who share various fractions of your genome, and it probably is something that health providers, employers, insurers, and even governments and the criminal justice system might want to (rightly or wrongly) know about. This raises complex ethical dilemas ${ }^{27-30}$. Even if genome data are anonymised (as they usually are in epidemiological studies), there is a risk of reidentification of persons based on their genome variation profile, and/or their phenotype and environmental profiles ${ }^{31}$. Exemplifying this, a recent paper has shown that an individual's involvement in a genetic study can be reliably established from just summary level allele frequency data (i.e., no individual genotypes) if this is available for two matched sample groups (such as GWAS cases and controls), and if the genome profile of the subject of interest is known ${ }^{32}$. A full discussion of the myriad questions surrounding 'data sensitivity' are beyond the scope of this review (see ref. 33), but a few points are worthy of mention. Currently, most databases try to avoid showing sufficient genotype or phenotype information to enable re-identification. This may not, however, be as easy as it seems - for example, in LSDBs where rare mutations/diseases may be reported along with geographical data. When G2P data do raise the possibility of reidentification, the current default position is to not make it publicly available, and pass access requests to the original custodians of the information. This stance was immediately adopted for summary level allele frequency datasets once it became clear that they could be used for individual identification ${ }^{34}$. But when even summary level data cannot be shared for unfettered research access, maybe it is time to start questioning when and where the protection of an individual's privacy becomes overly paranoid or too onerous to implement, given that it detracts from the wider research benefits of making data freely available? If, in reality, it will not be possible to completely ensure the anonymity of all research participants, then perhaps the optimal way forward may be to accept this, to make data more freely available, and concentrate instead on preventing and punishing abuse of the data. Given the existence of such perplexing privacy issues, an ethics advisory voice should arguably be an integral part of every G2P database.

\section{The Future: The Untapped Power of Federation}

Given the above considerations, federated databases can be expected to play an increasingly large role in the future of G2P information management. Before we discuss that role in more depth, it is useful to reflect on extreme versions of the federated and centralized models (Figure 1). The fully centralized model would involve all generated information being automatically piped into one large data center, from where all search and presentation activities are managed. A completely federated solution would involve all the domains' information being organized into geographically discrete packets (databases), with no regular data flows between them. Global searches would be mediated by portals that scan all available contents, and data presentation would be powered by each database for each item of its own content. Neither of these extremes is a realistic option for the G2P domain, due to the limitations of each model as described below, and a hybrid model would seem to offer the best way forward. Until now, however, most successful databases have been based on the centralized model. 
This likely reflects the newness of a field that only came into being with the emergence of the internet, and the fact that the current pressing need for more advanced solutions is relatively recent. Now, as internet and database technologies rapidly advance, federated systems are emerging alongside and intermingled with the existing established central databases.

Both central and federated systems have advantages and disadvantages. The main advantages of central databases include cost efficiency due to economies of scale, ease of management, and reliable archiving of the community's data. In contrast, federated databases represent a more complicated solution in terms of the required technologies, but they bring certain advantages that cannot be endowed by a central database (as detailed below). In large part this relates to 'ownership' and accreditation for the database teams, with the potential result that more and higher quality data can be gathered in a federated system, due to the reward gained by the workers involved. Federated and central database systems both provide centralized search capabilities, although federated alternatives can also offer more sophisticated search options via direct interrogation of the source databases.

Taking into account the challenges facing the G2P field as outlined above, and the pros and cons of each model, it seems evident that a purely centralized model cannot fulfill all the requirements of an optimal G2P databasing solution, and that some degree of federation is critical to the success of this enterprise. So what would a group of databases need to do to become usefully federated to an optimum degree? The first decision would involve the level of federation to be achieved. Essentially, this equates to deciding what portion of their content each database would wish to make available for other computers to read over the internet. They might choose to provide none, and instead transfer to one or more common search centers some pre-agreed 'core' data elements for each record they hold, along with links back to those entries in their database. The search system would then use that assemblage of minimal data items to enable multi-database searches, and report search results as a series of annotated links pointing back to the source databases. This partly centralized and partly federated solution is being piloted by several closely collaborating initiatives as a way to begin federating LSDBs ${ }^{35}$. Alternatively, and with more effort, the search platform could itself go and get the full details for each record of interest from the different sources (perhaps even by 'screen-scraping' if necessary) and compile this into a uniform dataset for presentation.

A more elegant way of federating would involve making some or all of the record details from each remote database directly searchable by other computers. This approach removes the need for sending in and regularly updating core datasets, thereby ensuring that searches through the central portal always query the very latest datasets. It also addresses the scalability problems outlined in the previous section, since any new LSDB merely needs to register its existence with the central portal to become part of the multi-database search catalogue. Another advantage is that it minimizes the workload of the central search system, as it no longer has to chase up and manage ever-changing core datasets every 24 hours or so. Finally, it alleviates many of the data complexity 
issues faced by central databases, in that each nodal database can provide and customize (at the final display stage) whatever additional record details it deems appropriate above and beyond the common data items made available as part of the federated search. Achieving this 'complete' federation, however, requires all participating databases to accept certain rules of the engagement. For example, the level of autonomy that each team can enjoy, in terms of database design, system execution, and the degree of association with the rest of the federation, must not be so high as to make the whole federation ineffective. Furthermore, all nodal databases must either adhere to certain standards so that their records can be easily integrated with those of others, or place advanced 'translation' software on top of their database so that search requests and result datasets can be freely communicated between remote and local computers.

Finally, certain other advantages of federation are also worthy of specific comment. The first relates to empowering and rewarding database creators (Figure 3). It takes effort to design, build, fund and continuously manage and curate a database - and it is all too often a thankless task. The federated model, however, places a lot more control and recognition in the hands of those running the individual databases. Federated databases have complete control over what records, and what details per record, are made available to different users at any point in time. This may be very important in the case of commercial databases, and it is highly important in the context of data sensitivity as mentioned above. Second, the federated structure distributes data management and curation work among many individuals, making the most of the expert knowledge of these individuals. A third advantage is that the federated structure enables new search portals to be set up quickly and easily, potentially offering unique new perspectives - for example, a gene-centric view for researchers specializing in a single gene, a disease-centric view for clinicians, a genome browser-based view for genomics researchers. Fourth, federated networks by default operate as democracies, so unilateral changes cannot be imposed on common aspects of the federated system (e.g., data models). This does not mean that innovation becomes stifled, but rather that new ideas will be widely debated, piloted and validated before they are implemented.

\section{The G2P Database Network}

Today, the components that are needed to create a powerful and highly integrated system, based upon a partially federated and partially centralized model, are either already available or in advanced stages of development. The key missing ingredients needed to bring the G2P network to life relate to expanding technology awareness, establishing recognition and reward systems, and targeting the appropriate allocation of sufficient funding. In Europe, many of these issues are being tackled head-on by initiatives such as the GEN2PHEN project(http://www.gen2phen.org), whilst consortia such as BBMRI (http://www.biobanks.eu) and ELIXIR (http://www.elixir-europe.org) are actively planning for the investment of up to several billion Euro into bioscience database and biobanking infrastructures (see also Table 1). 
Various technologies, such as web services and ontologies, that will underpin future G2P databasing have been discussed elsewhere ${ }^{36,23}$ (see Box 1 for a summary of the main components). Most importantly, the field will have to become increasingly standardized in order for a global network of G2P databases to inter-operate effectively. This standardization concern matters of both syntax (how data is organized) and semantics (the meaning of each data item).

A core syntax challenge involves designing and validating robust data models for different biomedical domains such that the models are inter-compatible. Typically, these models are also accompanied by standard specifications for data exchange formats, providing a basis for data exchange between systems. Various existing data models are currently being cross-compared and harmonised to enable more widespread data integration within a research domain, and even across different domains (see Box 2).

Semantic challenges involve ensuring that data items are represented in a way that conveys the same meaning to each and every person (or computer) that reads them. For example, a field named 'sample' may mean 'blood sample' in one database, but be taken to mean 'an individual sampled from a population' in another database. The goal is to structure and specify all of this in 'ontologies', and to build software and support systems that ensure the terms are used correctly ${ }^{37}$. Semantic standardization is difficult to achieve ${ }^{38}$, and tackling this issue across all bioscience sub-fields involves precisely defining a complete domain lexicon. To break this mammoth task down, researchers have set about working on ontologies for clearly demarcated subjects (such as 'Gene', 'DNA sequence' or 'anatomy'). A large and highly collaborative network of ontology groups has now grown into the Open Biomedical Ontologies (OBO) consortium ${ }^{39}$, which is currently tackling what may be the biggest ontology challenge of all: 'phenotypes'. Encouragingly, much progress has already been made on this undertaking, especially by the model organism database communities.

As syntactic and semantic standards start to fall into place, more groups are likely to start to consider building federated databases. Technologies will then be required that can broadcast, deliver, receive, or interrogate (locally or remotely) the available datasets. An early example of one such technology is the Distributed Annotation System (DAS) ${ }^{40}-$ a simple protocol for exchanging annotations on genomic sequences. Many databases already make their records available via their own DAS server to DAS clients such as the Ensembl browser. Those third-party datasets are then overlaid on other DAS-supplied information or locally available annotations, such as reference sets of genes - demonstrating the power of a federated system.

It is hoped that as databases, search platforms, visualization interfaces, and analysis tools start to become fully federated, and seamlessly joined together, the system will be transformed from a mere collection of cleverly connected data, into a universal G2P 'knowledge environment' - a place where new questions can be asked, new types of experiments can be performed in silico, and where new knowledge can be created. That, at least, is the vision of the 'semantic web' ${ }^{\text {'11 }}$, the proponents of which claim will comprise a highly-sophisticated and powerfully connected series of servers and client 
computers (the 'Grid') that together provide highly-automated data retrieval and analysis 'web-services' across the Internet. When this Grid-enabled G2P knowledge environment becomes a reality, we may find ourselves in a situation where the distinction between database entries and research manuscripts has become blurred, and where new paradigms like web publishing and real-time community markup of databased information are common place. Initial forays into this world of merged 'database-journal' publication vehicles are already taking place, one example being a partnership between the Human Genomics and Proteomics journal and the FINDbase database (http://www.sage-hindawi.com/journals/hgp/). Indeed, traditional journals may become a thing of the past, as they may well have evolved to become an integral part of this unified Grid of biomedical knowledge.

\section{Future challenges and opportunities}

Despite the optimistic future of G2P databases, a number of basic issues (mostly nontechnical ones) remain to be solved.

One of these involves getting enough people sufficiently well trained in the relevant technologies to build and connect all of the G2P databases. To achieve this, educators must decide to fund and organize such training. In parallel, software engineers can drastically reduce the level of competence required of the users by devising 'off the shelf' solutions. This philosophy lies at the heart of the GEN2PHEN project, which is producing empty 'database-in-a-box' installation packages along with training, and open-source complete genetic association database systems for download - all of which are based upon the PaGE-OM data model (http://www.pageom.org), giving them the option of federation.

Another issue is the question of tracking who is building G2P databases and populating them with useful data. Several initiatives have recently been launched to look into this. For example, the idea of 'microattribution' has been proposed whereby database systems would track the interest in each database record ${ }^{42}$, record this in relation to the original submitter of the record, and thereby steadily assemble a metric for the value of each person's database contributions. Similarly, database creators would be able to extrapolate from this kind of information something akin to a journal impact factor. This will require cooperation of journals, database creators and funders, all of whom would need to use an agreed tracking system. Additionally, success would depend upon there being a way to assign globally-unique identifiers (GUIDs) to individual data packets (e.g., database records) on the internet. Since the semantic web will also need such identifiers (not only for data, but also for services, concepts, metadata, etc) several solutions to this problem are now being evaluated. An alternative 'Bio-Resource Impact Factor' (BRIF) approach to accreditation has been proposed ${ }^{43}$, the scope of which would include G2P databases. BRIF is more directly akin to the journal Impact Factor. As journals are steadily overtaken by databases as the preferred means for getting data into the public domain, BRIF, or something like it, will be needed to demonstrate 
researchers' productivity and the importance of their work. It will also help in making it evident to funding bodies that database creation efforts are worthy of support $-\mathrm{a}$ message that presently needs some re-enforcement ${ }^{44}$.

Considerable sums of money are being spent on G2P research to improve our understanding of health and disease so that medical care will advance. It follows therefore that the G2P databases should remain tightly focused on the needs of the medical community. The problem, however, is that whereas imprecise knowledge and uncertain data are an essential part of research, the clinical world requires more straightforward, reproducible information upon which to base its decision making. Perhaps then, one of the real unmet challenges for G2P databases is that of distilling from basic research data the kinds of watertight conclusions and predictions that would help physicians diagnose a patient. Close attention needs to be paid to how this information is presented, as researchers and clinicians typically have very different expectations when searching for information. These important issues are well known to those who work at the interface of bioinformatics and medical informatics. Examples of key efforts designed to close the gap between the two include the development of electronic healthcare records (EHR), and the work of the Health Level Seven (HL7) organization towards genomics standards in medical information.

In summary, the field of G2P databasing is at a significant stage in its development, taking into account lessons from the past, and being challenged by the exponentially growing and rapidly changing datasets of the present and the future. In this review, we have tried to cover the technical solutions and the logical ways forward for the field, all of which are buttressed by extensive open-source collaboration and contribute to the emerging 'cyberinfrastructure for the biological sciences' ${ }^{45}$. We can therefore expect G2P databasing to advance significantly in the near future, enabling research and clinical practitioners to make the very best use of the wealth of G2P data now being generated. 


\section{Figure Legends}

Figure 1: Extreme Models for Database Integration

Radical forms of centralized and federated database networks are illustrated on either side of this image. In the centralized model on the left, outstations or 'nodes' (small circles) merely gather and prepare data for transfer to a massive central 'hub' (large circle), where it is stored, integrated, and made available for searching and presentation. In the federated model on the right, the outstations are replaced by fullyfunctional databases (the eight medium sized circles) that gather and expertly curate data, provide various means for human and machine based searching/accessing of this information, and offer a range of data presentation options. In this latter model, the hub receives no data from the nodes, but undertakes the important job of coordinating the nodes and brokering searches across the other databases. The genotype-to-phenotype database network of the future will probably be based upon a hybrid of these two extreme models.

\section{Figure 2: Databases and Database Networks}

Early genotype-to-phenotype (G2P) databases were based upon many different designs (indicated by differentially colored circles) with very few connections between them (indicated by lines joining the circles). As the field develops, databases will instead be built upon more standardized design and operation principles, enabling extensive interconnectivity between projects. Each resource in the resulting network may have an emphasis upon being a data storage 'node' (smaller circles) or a data searching 'hub' (larger circles), or a combination of the two. It is hoped that emerging semantic web technologies will develop the network further into a powerful and seamless G2P knowledge environment.

\section{Figure 3: Success Depends Upon Recognition and Reward}

The utility of any future genotype-to-phenotype database network and its supporting infrastructure will depend upon how effectively information gets into that system. Individuals responsible for establishing and operating this 'data flow' - from the wet bench scientist that produces the raw data, right through to the people that make the integrated datasets available for searching and access - will all need to be recognized, rewarded, and thereby motivated to play their part. Mechanisms for achieving this in the context of databases (as opposed to data publication via journals) are yet to be put in place. 


\section{Box 1: Technologies in Genotype-to-Phenotype Databasing}

Databases and database networks will be key to organizing, storing, and providing access to the wealth of biomedical data already produced and yet to be generated. The task of building the necessary databases is primarily a technological construction effort, since the required technology solutions are already well developed or at least identified in principle. Some of the core concepts behind these technologies are listed below:

Object (Data) model - A formalized conceptualization of how data elements, or objects, are structured and organized, and how they are connected to other data elements. This may include semantic information on those objects and connections, by way of references to ontologies (see below). An example is the Microarray Gene Expression Object mode ${ }^{46}$ (MAGE-OM) which standardizes the representation of micro-array information, spanning experiment design and data.

Exchange format - The specifications of the syntax, or physical representation, of data complying with the model. This is essential for unambiguous transmission of data between computers. Examples range from the simple FASTA format used to exchange DNA and protein sequence data, through to the elaborate XML-based MAGE-ML for MAGE-OM compliant microarray data.

Ontology - A controlled vocabulary of terms for concepts, including their meaning and well-defined relationships between them. Ontologies enable the representation of domain-specific knowledge and, when used properly, make database searches far more powerful. Examples include Gene Ontology ${ }^{47}(\mathrm{GO})$ for annotating gene products from many species, and $\mathrm{FuGO}^{48}$ for functional genomics investigations.

Globally-unique identifier (GUID) - A digital object identifier, which is guaranteed to be unique and persistent across the intended usage domain. GUIDs solve data integration problems that result from ambiguity in names, or identity, of biological concepts and objects, such as genes and proteins. GUIDs are key component of Semantic Web technologies such as the Resource Description Framework (RDF:

http://www.w3.org/RDF). Examples currently being evaluated include Persistent URLs (http://www.purl.org) and Life Science Identifiers ${ }^{49}$ (LSIDs).

Web services - A series of standard protocols for facilitating machine-to-machine interaction over the Internet. Web services simplify the task of 'plumbing together' distributed data retrieval or analysis services over the network, forming the basis of the service-oriented architecture (SOA: http://www.w3.org/TR/2003/WD-ws-arch20030514/). An example of a service-oriented 'Grid' is provided by the National Cancer Institute's caGrid ${ }^{50}$. 


\section{Box 2: Harmonising Data Models}

Data models can often be aligned or 'mapped' to each other to identify similarities and differences. Once this is done, and equivalent concepts are relationships thereby identified, it is then possible to specify a consensus model and/or derive a data exchange format with which both models will be compatible.

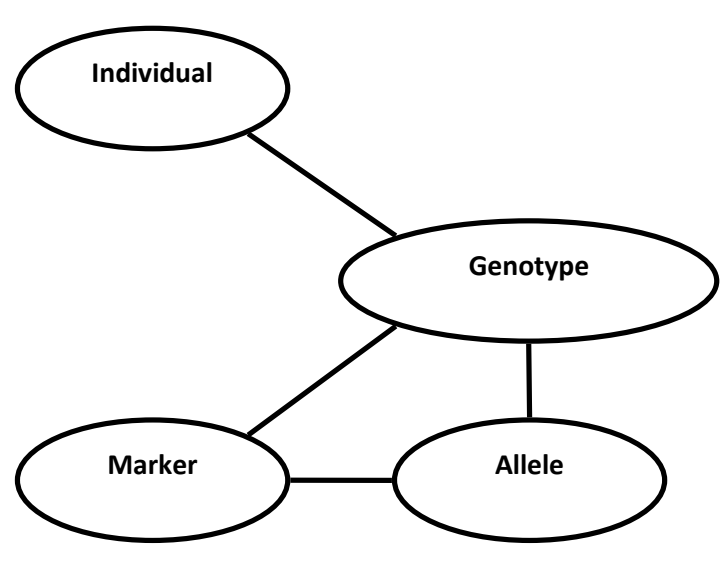

a) MIQAS

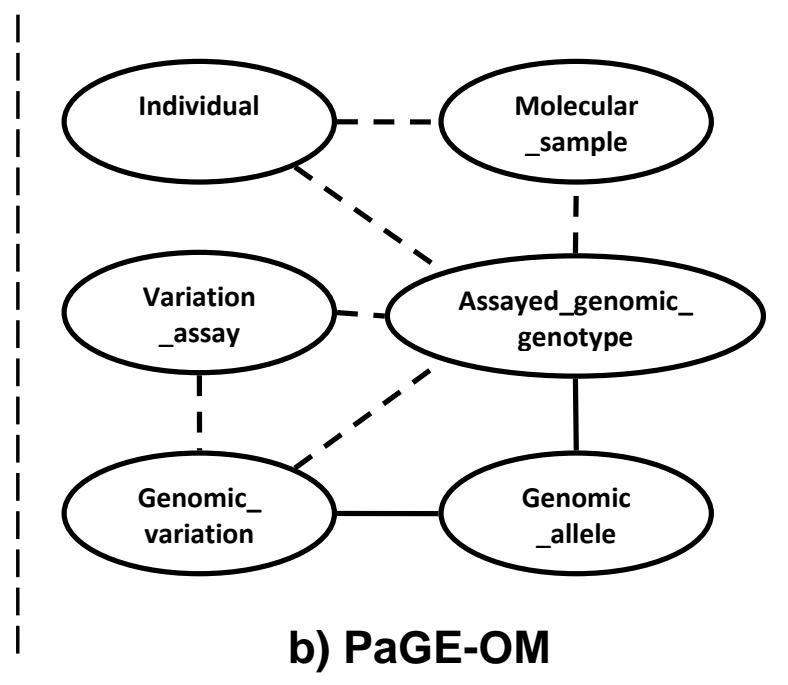

b) PaGE-OM

This is illustrated in the figure for sub-portions of two related data models, a) the Minimum Information for QTLs and Association Studies specification (MIQAS: http://miqas.sourceforge.net), and b) the Phenotype and Genotype Experiment Object Model (PaGE-OM: http://www.pageom.org). Equivalent entities in these models are in some cases named differently (e.g., 'Marker' and 'Genomic_variation'), and so to highlight the corresponding item pairs they have been placed in the same relative positions in the diagrams and are shown in the same colour. Naming discrepancies are problematic, especially when the same name is used to mean different things between models (e.g., 'Sample' for a person or a reagent). Such confusion is eliminated when models include semantic information on their components, by references to ontologies. Solid lines in the above illustrations indicate relationships (e.g., a 'Marker' has 'Alleles'), and the dotted lines in PaGE-OM indicate that relationships may be optional to allow for data elements that might not be known (e.g., the Variation_assay used in a genotyping experiment). Based upon such mapping diagrams, data exchange format can be specified that support only the common components from the models, or they may be extended to include some non-common items whereupon those data elements would be declared optional.

Similar inter-model mapping can be done between sub-domains where the underlying models may be quite different, so long as the models have at least some common concepts or attributes. Datasets produced according to those models may then be connected together, rather than fully merged, by linking through those common fields on data rows where the values are identical (e.g., a SNP marker identifier), as explained elsewhere ${ }^{51}$. 


\section{Table 1: Genotype-to-Phenotype Database Infrastructure and Coordination Projects}

\begin{tabular}{|c|c|}
\hline $\begin{array}{l}\text { BBMRI } \\
\text { (http://www.biobanks.eu/) }\end{array}$ & $\begin{array}{l}\text { ESFRI program for preparing to construct a pan-European } \\
\text { Biobanking and Biomolecular Resources Research } \\
\text { Infrastructure. An ESFRI project }\end{array}$ \\
\hline $\begin{array}{l}\text { caBIG } \\
\text { (https://cabig.nci.nih.gov/) }\end{array}$ & $\begin{array}{l}\text { The Cancer Biomedical Informatics Grid. Data integration } \\
\text { network and application infrastructure developed for the } \\
\text { cancer research community. }\end{array}$ \\
\hline $\begin{array}{l}\text { CASIMIR } \\
\text { (http://www.casimir.org.uk/) }\end{array}$ & $\begin{array}{l}\text { Coordination and Sustainability of International Mouse } \\
\text { Informatics Resources. EU funded project on co- ordination } \\
\text { and integration of mouse model organism databases. }\end{array}$ \\
\hline $\begin{array}{l}\text { EATRIS } \\
\text { (http://www.eatris.eu) }\end{array}$ & $\begin{array}{l}\text { The European Advanced Translational Research } \\
\text { Infrastructure in Medicine. An ESFRI project aimed at } \\
\text { translating research findings into improved diagnosis, } \\
\text { disease prevention, and treatment. }\end{array}$ \\
\hline $\begin{array}{l}\text { ECRIN } \\
\text { (http://www.ecrin.org/) }\end{array}$ & $\begin{array}{l}\text { European Clinical Research Infrastructures Network. An } \\
\text { ESFRI program aimed at integrating national clinical } \\
\text { research facilities into a pan-European infrastructure. }\end{array}$ \\
\hline $\begin{array}{l}\text { EGEE } \\
\text { (http://www.eu-egee.org/) }\end{array}$ & $\begin{array}{l}\text { Enabling Grids for E-sciencE. Large EU funded } \\
\text { multidisciplinary infrastructure project. }\end{array}$ \\
\hline $\begin{array}{l}\text { ELIXIR } \\
\text { (http://www.elixir-europe.org) }\end{array}$ & $\begin{array}{l}\text { European Life-science Infrastructure for Biological } \\
\text { Information. EU funded bioinformatics infrastructure } \\
\text { program for life science research. An ESFRI project. }\end{array}$ \\
\hline $\begin{array}{l}\text { EMBRACE } \\
\text { (http://www.embracegrid.info) }\end{array}$ & $\begin{array}{l}\text { European Model for Bioinformatics Research and } \\
\text { Community Education. Collaboration network in area of } \\
\text { Grid computing and databasing in biomolecular research. }\end{array}$ \\
\hline $\begin{array}{l}\text { ESFRI } \\
\text { (http://cordis.europa.eu/esfri/) }\end{array}$ & The European Strategy Forum on Research Infrastructures. \\
\hline $\begin{array}{l}\text { EUROGENETEST } \\
\text { (http://www.eurogentest.org/) }\end{array}$ & $\begin{array}{l}\text { EU-funded Network of Excellence fostering standardization } \\
\text { and harmonization of genetic testing across Europe } \\
\text { including informatics, ethics, new technologies, education } \\
\text { and quality management. }\end{array}$ \\
\hline $\begin{array}{l}\text { GEN2PHEN } \\
\text { (http://www.gen2phen.org) }\end{array}$ & $\begin{array}{l}\text { Genotype to Phenotype databases. An EU funded project } \\
\text { aiming to unify human and model organism G2P databases } \\
\text { towards increasingly holistic views into this information. }\end{array}$ \\
\hline $\begin{array}{l}\text { GMOD } \\
\text { (http://gmod.org) }\end{array}$ & $\begin{array}{l}\text { Generic Model Organism Database project. A collection of } \\
\text { open source software tools for creating and managing } \\
\text { genome-scale biological databases. }\end{array}$ \\
\hline $\begin{array}{l}\text { HL7 } \\
\text { (http://www.hl7.org/) }\end{array}$ & $\begin{array}{l}\text { Standardization organization operating on healthcare arena. } \\
\text { HL7 V3 is messaging standard developed by the HL7 for } \\
\text { health care domain }\end{array}$ \\
\hline $\begin{array}{l}\text { HuGENet } \\
\text { (http://www.cdc.gov/genomics/hugenet/) }\end{array}$ & $\begin{array}{l}\text { Human Genome Epidemiology Network. International } \\
\text { collaboration of individuals and organization in field of } \\
\text { genetic epidemiology. }\end{array}$ \\
\hline $\begin{array}{l}\text { HVP } \\
\text { (http://www.humanvariomeproject.org/) }\end{array}$ & $\begin{array}{l}\text { An open organisation aiming to help catalogue all human } \\
\text { Mendelian genetic variation, making that information freely } \\
\text { available to researchers, clinicians and patients worldwide. }\end{array}$ \\
\hline MIBBI ${ }^{52}$ (http://www.mibbi.org) & $\begin{array}{l}\text { Minimum Information for Biological and Biomedical } \\
\text { Investigations is a project and web resource promoting the } \\
\text { development and use of minimum information specifications } \\
\text { and checklists. }\end{array}$ \\
\hline $\begin{array}{l}\text { OBIBA } \\
\text { (http://www.obiba.org/) }\end{array}$ & $\begin{array}{l}\text { Obiba is an open source project whose aim is to build an } \\
\text { open software infrastructure applications and software } \\
\text { components for biobanking. One of the P3G core projects. }\end{array}$ \\
\hline
\end{tabular}




\begin{tabular}{|l|l|}
\hline $\begin{array}{l}\text { OBO } \\
\text { (http://obofoundry.org/) }\end{array}$ & $\begin{array}{l}\text { The Open Biomedical Ontologies. International community } \\
\text { for supporting development and use of ontologies in } \\
\text { biomedical domain. }\end{array}$ \\
\hline $\begin{array}{l}\text { OpenEHR } \\
\text { (http://www.openehr.org) }\end{array}$ & $\begin{array}{l}\text { Data standards and modeling framework for managing } \\
\text { electronic health care data. }\end{array}$ \\
\hline $\begin{array}{l}\text { P3G } \\
\text { (http://www.p3gconsortium.org/) }\end{array}$ & $\begin{array}{l}\text { The Public Population Project in Genomics. An international } \\
\text { consortium promoting collaboration between population } \\
\text { genomics researchers. The P3G observatory } \\
\text { (http://www.p3gobservatory.org) provides a central } \\
\text { repository of relevant tools and information. }\end{array}$ \\
\hline
\end{tabular}




\section{References}

1. Wheeler, D.L. et al. Database resources of the National Center for Biotechnology Information. Nucleic Acids Res 35, D5-12(2007).

2. Hubbard, T. et al. The Ensembl genome database project. Nucleic Acids Res 30, 38-41(2002).

3. Kent, W.J. et al. The Human Genome Browser at UCSC. Genome Res. 12, 996-1006(2002).

4. Stein, L. Creating a bioinformatics nation. Nature 417, 119-120(2002).

5. Miyazaki, S. et al. DDBJ in the stream of various biological data. Nucleic Acids Research 32, D31-34(2004).

6. Benson, D.A. et al. GenBank. Nucleic Acids Research 36, D25-30(2008).

7. Kanz, C. et al. The EMBL Nucleotide Sequence Database. Nucleic Acids Research 33, D29-33(2005).

8. Chen, N. et al. WormBase: a comprehensive data resource for Caenorhabditis biology and genomics. Nucleic Acids Res 33, D383-389(2005).

9. Twigger, S.N. et al. The Rat Genome Database, update 2007-easing the path from disease to data and back again. Nucleic Acids Res 35, D658-662(2007).

10. Bult, C.J. et al. The Mouse Genome Database (MGD): mouse biology and model systems. Nucleic Acids Res 36, D724-728(2008).

11. Hamosh, A. et al. Online Mendelian Inheritance in Man (OMIM), a knowledgebase of human genes and genetic disorders. Nucleic Acids Res 33, D514-517(2005).

12. McKusick, V.A. Mendelian Inheritance in Man. A Catalog of Human Genes and Genetic Disorders. (Johns Hopkins University Press: 1966).

13. Ball, E.V. et al. Microdeletions and microinsertions causing human genetic disease: common mechanisms of mutagenesis and the role of local DNA sequence complexity. Hum Mutat 26, 205-213(2005).

14. Altman, R.B. PharmGKB: a logical home for knowledge relating genotype to drug response phenotype. Nat Genet 39, 426-426(2007).

15. Lehmann, H. \& Kynoch, P.A.M. Human haemoglobin variants and their characteristics. (NorthHolland Publishing: Amsterdam, 1976).

16. Horaitis, O. et al. A database of locus-specific databases. Nat Genet 39, 425(2007). 
17. Mailman, M.D. et al. The NCBI dbGaP database of genotypes and phenotypes. Nat Genet 39, 1181-1186(2007).

18. Becker, K.G. et al. The Genetic Association Database. Nat Genet 36, 431-432(2004).

19. Bertram, L. et al. Systematic meta-analyses of Alzheimer disease genetic association studies: the AlzGene database. Nat Genet 39, 17-23(2007).

20. Allen, N.C. et al. Systematic meta-analyses and field synopsis of genetic association studies in schizophrenia: the SzGene database. Nature Genetics 40, 827-834(2008).

21. Mardis, E.R. The impact of next-generation sequencing technology on genetics. Trends Genet 24, 133-141(2008).

22. Howe, D. et al. Big data: The future of biocuration. Nature 455, 47-50(2008).

23. Goble, C. \& Stevens, R. State of the nation in data integration for bioinformatics. J Biomed Inform. 41, 687-93(2008)

24. Knoppers, B. et al. Population Genomics: The Public Population Project in Genomics (P(3)G): a proof of concept? Eur J Hum Genet 16, 664-665(2008).doi:10.1038/ejhg.2008.55

25. Ioannidis, J.P.A. et al. A road map for efficient and reliable human genome epidemiology. Nat Genet 38, 3-5(2006).

26. Elnitski, L.L. et al. The ENCODEdb portal: simplified access to ENCODE Consortium data. Genome Res 17, 954-959(2007).

27. Hoyweghen, I.V. \& Horstman, K. European practices of genetic information and insurance: lessons for the Genetic Information Nondiscrimination Act. JAMA 300, 326-7(2008).

28. Diergaarde, B. et al. Genetic information: Special or not? Responses from focus groups with members of a health maintenance organization. Am J Med Genet A 143, 564-9(2007).

29. Gilbar, R. Patient autonomy and relatives' right to know genetic information. Medicine and law 26, 677-97(2007).

30. Knoppers, B.M. et al. The emergence of an ethical duty to disclose genetic research results: international perspectives. Eur J Hum Genet 14, 1170-8(2006).

31. Godard, B. et al. Data storage and DNA banking for biomedical research: informed consent, confidentiality, quality issues, ownership, return of benefits. A professional perspective. Eur J Hum Genet 11 Suppl 2, S88-122(2003).

32. Homer, N. et al. Resolving individuals contributing trace amounts of DNA to highly complex mixtures using high-density SNP genotyping microarrays. PLoS Genet 4, e1000167(2008). 
33. Cambon-Thomsen, A., Rial-Sebbag, E. \& Knoppers, B.M. Trends in ethical and legal frameworks for the use of human biobanks. Eur Respir J 30, 373-382(2007).

34. Zerhouni, E.A. \& Nabel, E.G. Protecting aggregate genomic data. Science 322, 44(2008).

35. Giardine, B. et al. PhenCode: connecting ENCODE data with mutations and phenotype. Hum Mutat 28, 554-562(2007).

36. Stein, L.D. Integrating biological databases. Nat Rev Genet 4, 337-345(2003).

37. Stevens, R., Goble, C.A. \& Bechhofer, S. Ontology-based knowledge representation for bioinformatics. Brief Bioinform 1, 398-414(2000).

38. Quackenbush, J. Standardizing the standards. Mol Syst Biol 2, 2006.0010(2006).

39. Smith, B. et al. The OBO Foundry: coordinated evolution of ontologies to support biomedical data integration. Nat Biotechnol 25, 1251-1255(2007).

40. Dowell, R.D. et al. The Distributed Annotation System. BMC Bioinformatics 2, 7(2001).

41. Berners-Lee, T., Hendler, J. \& Lassila, O. The Semantic Web - A new form of Web content that is meaningful to computers will unleash a revolution of new possibilities. Scientific American 284, 34-43(2001).

42. Editors Compete, collaborate, compel. Nature Genetics 39, 931(2007).

43. Kauffmann, F. \& Cambon-Thomsen, A. Tracing biological collections: between books and clinical trials. JAMA 299, 2316-2318(2008).

44. Merali, Z. \& Giles, J. Databases in peril. Nature 435, 1010-1011(2005).

45. Stein, L.D. Towards a cyberinfrastructure for the biological sciences: progress, visions and challenges. Nat Rev Genet 9, 678-88(2008).

46. Spellman, P.T. et al. Design and implementation of microarray gene expression markup language (MAGE-ML). Genome Biol 3, research0046.1-0046.9(2002).

47. The Gene Ontology Consortium. Gene Ontology: tool for the unification of biology. Nat Genet 25, 25-29(2000).

48. Jones, A.R. et al. The Functional Genomics Experiment model (FuGE): an extensible framework for standards in functional genomics. Nat Biotechnol 25, 1127-1133(2007).

49. Clark, T., Martin, S. \& Liefeld, T. Globally distributed object identification for biological knowledgebases. Brief Bioinform 5, 59-70(2004).

50. Saltz, J. et al. caGrid: design and implementation of the core architecture of the cancer biomedical informatics grid. Bioinformatics 22, 1910-1916(2006). 
51. Wang, X., Gorlitsky, R. \& Almeida, J.S. From XML to RDF: how semantic web technologies will change the design of 'omic' standards. Nat Biotechnol 23, 1099-1103(2005).

52. Taylor, C.F. et al. Promoting coherent minimum reporting guidelines for biological and biomedical investigations: the MIBBI project. Nat Biotechnol 26, 889-896(2008).

\section{Acknowledgements}

The authors acknowledge the valuable ideas, advice, and funding, provided by the GEN2PHEN project as part of the European Community's Seventh Framework Programme (FP7/2007-2013) under grant agreement number 200754, that enabled the preparation of this review. 
Figure 1

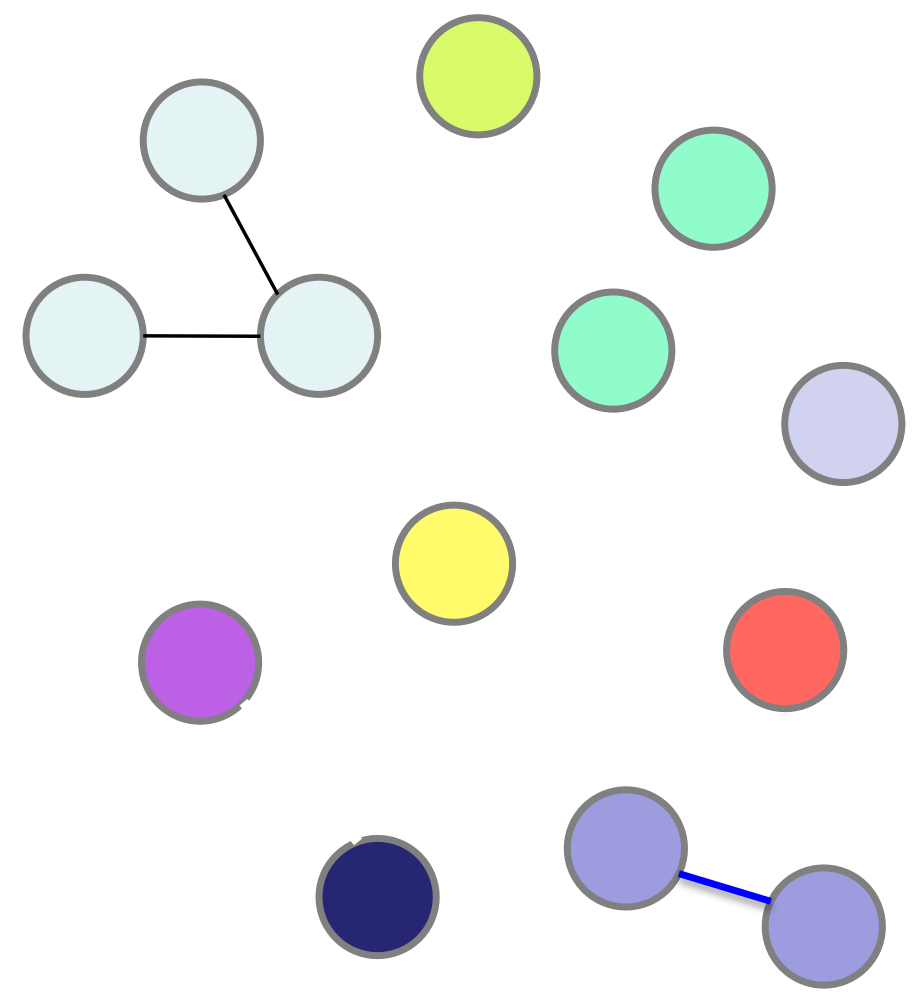

Current Databases; disparate silos

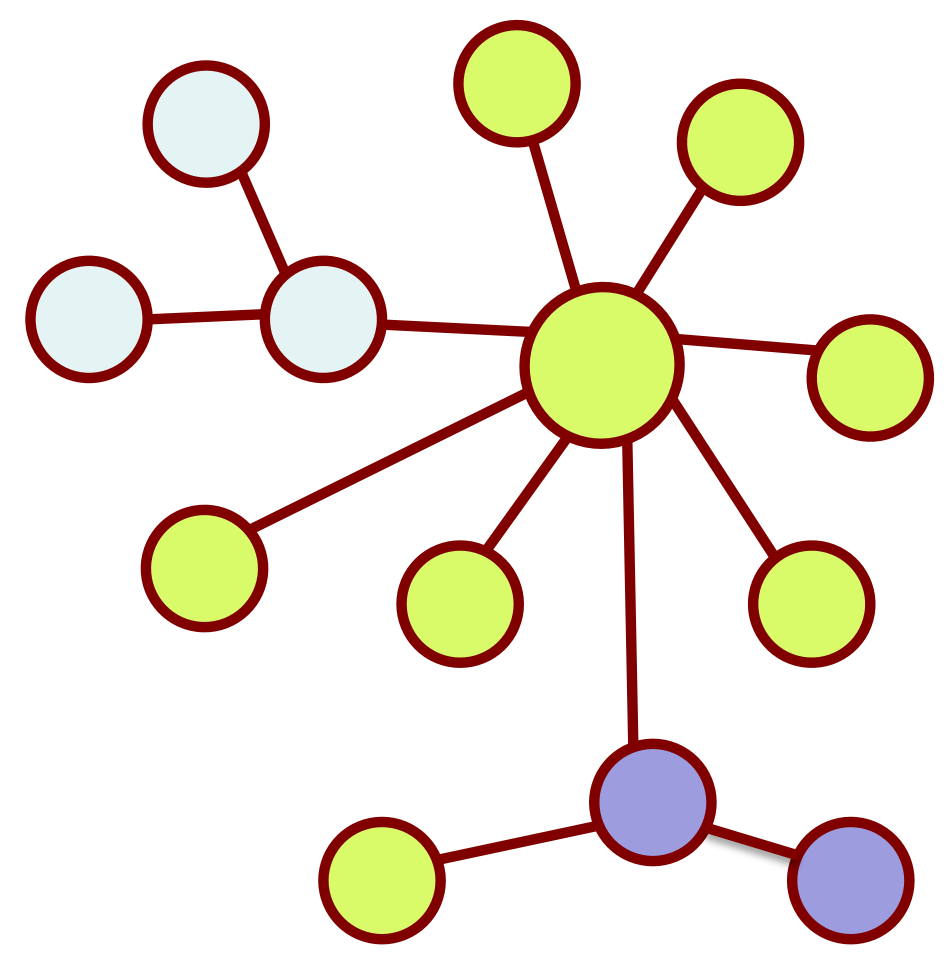

Future databases; an inter-connected network 
Figure 2

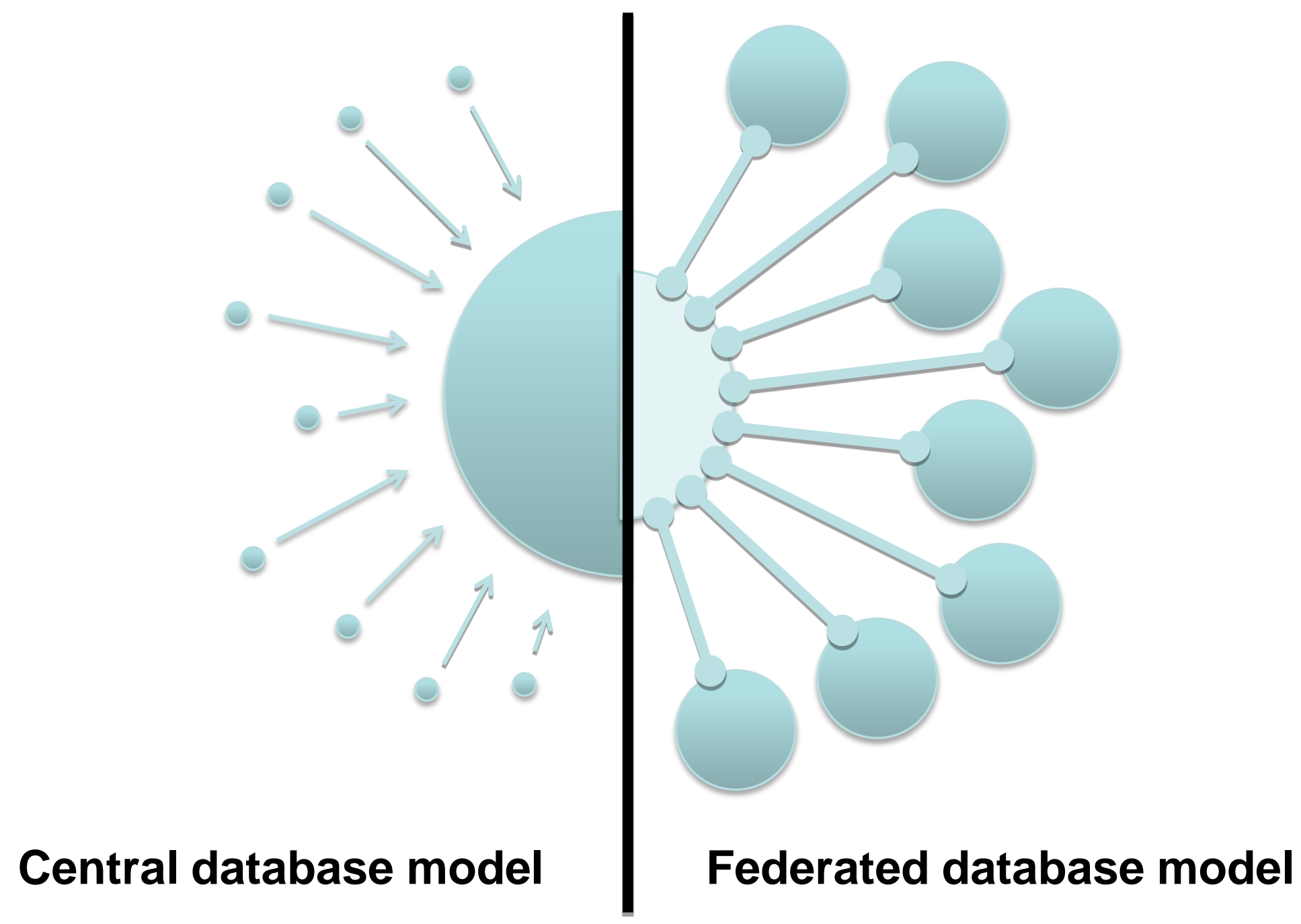


